\title{
Research on Consumers’ Purchasing Behavior and Marketing Strategy Based on Mobile Internet Environment
}

\author{
Du Wei \\ Hainan Software Technology College, Qionghai, Hainan, China \\ 1258502015@qq.com
}

Keywords: mobile Internet environment; consumers’ purchasing behavior; research on marketing strategy

\begin{abstract}
The changes in China's economic system boost the Internet and lay a solid foundation for the development of the network economy. This paper analyzes the consumers' purchasing behavior and marketing strategy based on mobile internet environment.
\end{abstract}

\section{Introduction}

The changes in China's economic system boost the Internet and lay a solid foundation for the development of the network economy. The network economy has changed the relationship between companies, consumers, and enterprises. This paper analyzes the consumers' purchasing behavior and marketing strategy based on mobile internet environment.

\section{Model analysis of consumers' purchasing behavior and marketing strategy based on mobile internet environment}

\subsection{Model}

This paper takes the mobile Internet as a research case to explore the empirical research on the relationship between internet interactive marketing strategy and consumer behavior, including mobile QR code strategy, message website strategy, mobile search strategy, mobile business district strategy, wireless website strategy and Bluetooth interaction strategy

\subsection{Consumption}

Under the mobile internet interactive marketing model, companies can enhance interactive marketing with consumers and increase consumers' satisfaction. As methods of improving interactive marketing, strategies of message website, mobile search, mobile business district, wireless website and Bluetooth interaction can affect consumers' satisfaction. Based on the above analyses, the following consumptions are proposed in this paper.

Mobile Internet Enterprises:

$\mathrm{H}_{1}$ : There is a positive correlation between interactive marketing strategies and consumer satisfaction.

$\mathrm{H}_{11}$ : There is a positive correlation between message website marketing strategies and consumer satisfaction.

$\mathrm{H}_{12}$ : There is a positive correlation between mobile search marketing strategies and consumer satisfaction.

$\mathrm{H}_{13}$ : There is a positive correlation between mobile business district marketing strategies and consumer satisfaction.

$\mathrm{H}_{14}$ : There is a positive correlation between wireless website marketing strategies and consumer satisfaction.

$\mathrm{H}_{15}$ : There is a positive correlation between Bluetooth interaction marketing strategies and consumer satisfaction. 
$\mathrm{H}_{16}$ : There is a positive correlation between QR code scanning marketing strategies and consumer satisfaction.

Based on the above analyses, this paper proposes the following consumptions:

$\mathrm{H}_{2}$ : By improving the consumer satisfaction, Interactive marketing strategies are positively correlated to consumers' behavior.

$\mathrm{H}_{21}$ : By improving the consumer satisfaction, message website marketing strategies are positively correlated to consumers' behavior.

$\mathrm{H}_{22}$ : By improving the consumer satisfaction, mobile search marketing strategies are positively correlated to consumers' behavior.

$\mathrm{H}_{23}$ : By improving the consumer satisfaction, mobile business district marketing strategies are positively correlated to consumers' behavior.

$\mathrm{H}_{24}$ : By improving the consumer satisfaction, wireless website marketing strategies are positively correlated to consumers' behavior.

$\mathrm{H}_{25}$ : By improving the consumer satisfaction, Bluetooth interaction marketing strategies are positively correlated to consumers' behavior.

$\mathrm{H}_{26}$ : By improving the consumer satisfaction, QR code scanning strategies are positively correlated to consumers' behavior.

\subsection{Variable Selection}

There are three main variables involved in the model. The first is the mobile internet interactive marketing strategy, consumer satisfaction and consumer behavior. Among them, the Internet interactive marketing strategy includes six aspects of measurement: message website strategy, mobile search strategy, mobile business district strategy, wireless website strategy and Bluetooth interaction strategy. The meaning of the variables is shown in Table 1.

Table 1 Meaning of variables

\begin{tabular}{|c|c|c|}
\hline Variables & Dimension & Basic meaning \\
\hline \multirow{6}{*}{$\begin{array}{l}\text { Mobile } \\
\text { internet } \\
\text { interactive } \\
\text { marketing } \\
\text { strategy }\end{array}$} & $\begin{array}{l}\text { Message } \\
\text { website } \\
\text { strategy }\end{array}$ & $\begin{array}{l}\text { The number of mobile internet companies marketing by SMS } \\
\text { messaging and wap addressing within one month }\end{array}$ \\
\hline & $\begin{array}{l}\text { Mobile search } \\
\text { strategy }\end{array}$ & $\begin{array}{l}\text { The number of data messages that you have searched using SMS, } \\
\text { WAP, and IVR within one month }\end{array}$ \\
\hline & $\begin{array}{c}\text { Mobile } \\
\text { business } \\
\text { district } \\
\text { strategy }\end{array}$ & $\begin{array}{l}\text { The number of visits you make into the hall using mobile virtual } \\
\text { business within one month }\end{array}$ \\
\hline & $\begin{array}{l}\text { Wireless } \\
\text { website } \\
\text { strategy }\end{array}$ & $\begin{array}{l}\text { The number of times you received marketing pushes through your } \\
\text { corporate wireless site within one month }\end{array}$ \\
\hline & $\begin{array}{l}\text { Bluetooth } \\
\text { interaction } \\
\text { strategy }\end{array}$ & $\begin{array}{l}\text { The number you have interacted with mobile internet company via } \\
\text { Bluetooth technology within one month }\end{array}$ \\
\hline & $\begin{array}{l}\text { OR code } \\
\text { scanning by } \\
\text { phone }\end{array}$ & $\begin{array}{l}\text { The number you have interacted with mobile internet companies by } \\
\text { QR code scanning of phone within one month }\end{array}$ \\
\hline \multirow{2}{*}{\multicolumn{2}{|c|}{ Consumer satisfaction }} & $\begin{array}{l}\text { The expected value of the goods or services that you purchase from } \\
\text { the mobile Internet are consistent with the actual value }\end{array}$ \\
\hline & & $\begin{array}{l}\text { You are very impressed with goods or services that you purchase } \\
\text { from the mobile internet }\end{array}$ \\
\hline \multirow{2}{*}{\multicolumn{2}{|c|}{ Consumer satisfaction }} & $\begin{array}{l}\text { You will continue to purchase goods or services from this mobile } \\
\text { internet platform }\end{array}$ \\
\hline & & $\begin{array}{l}\text { You will recommend goods or services on this mobile internet } \\
\text { platform to others }\end{array}$ \\
\hline
\end{tabular}


Table 2 Correlation Test between Interactive Marketing and Consumer Behavior in Mobile Internet

\begin{tabular}{|c|c|c|c|c|c|c|c|c|c|}
\hline & $\begin{array}{c}\text { Internet } \\
\text { interactive } \\
\text { marketing } \\
\text { strategy }\end{array}$ & $\begin{array}{l}\text { Message } \\
\text { website } \\
\text { strategy }\end{array}$ & $\begin{array}{l}\text { Mobile } \\
\text { search } \\
\text { strategy }\end{array}$ & $\begin{array}{c}\text { Mobile } \\
\text { business } \\
\text { district } \\
\text { strategy }\end{array}$ & $\begin{array}{l}\text { Wireless } \\
\text { website } \\
\text { strategy }\end{array}$ & $\begin{array}{c}\text { Bluetooth } \\
\text { interaction } \\
\text { strategy }\end{array}$ & $\begin{array}{l}\text { QR code } \\
\text { scanning } \\
\text { by phone }\end{array}$ & $\begin{array}{c}\text { Consumer } \\
\text { satisfaction }\end{array}$ & $\begin{array}{c}\text { Behavior } \\
\text { of } \\
\text { consumers }\end{array}$ \\
\hline $\begin{array}{c}\text { Internet } \\
\text { interactive } \\
\text { marketing } \\
\text { strategy }\end{array}$ & 1 & & & & & & & & \\
\hline $\begin{array}{l}\text { Message } \\
\text { website } \\
\text { strategy }\end{array}$ & 0.455 & 1 & & & & & & & \\
\hline $\begin{array}{l}\text { Mobile } \\
\text { search } \\
\text { strategy }\end{array}$ & 0.165 & 0.254 & 1 & & & & & & \\
\hline $\begin{array}{l}\text { Mobile } \\
\text { business } \\
\text { district } \\
\text { strategy }\end{array}$ & 0.455 & 0.211 & 0.325 & 1 & & & & & \\
\hline $\begin{array}{l}\text { Wireless } \\
\text { website } \\
\text { strategy }\end{array}$ & 0.235 & 0.322 & 0.022 & & 1 & & & & \\
\hline $\begin{array}{c}\text { Bluetooth } \\
\text { interaction } \\
\text { strategy }\end{array}$ & 0.515 & 0.122 & 0.354 & 0.015 & 0.205 & 1 & & & \\
\hline $\begin{array}{c}\text { QR code } \\
\text { scanning by } \\
\text { phone }\end{array}$ & 0.218 & 0.3456 & 0.265 & 0.21 & 0.454 & 0.125 & 1 & & \\
\hline $\begin{array}{l}\text { Consumer } \\
\text { satisfaction }\end{array}$ & 0.365 & 0.596 & 0.122 & 0.012 & 0.214 & 0.012 & 0.332 & 1 & \\
\hline $\begin{array}{c}\text { Behavior of } \\
\text { consumers }\end{array}$ & 0.111 & 0.354 & 0.545 & 0.035 & 0.318 & 0.941 & 0.255 & 0.023 & 1 \\
\hline
\end{tabular}

Table 3 Test for stationarity of variables

\begin{tabular}{|c|c|c|c|c|c|}
\hline Original variable & ADF Value & $\begin{array}{c}5 \% \text { critical } \\
\text { value }\end{array}$ & $\begin{array}{c}\text { first order difference } \\
\text { variable }\end{array}$ & ADF Value & $\begin{array}{c}5 \% \text { critical } \\
\text { value }\end{array}$ \\
\hline $\begin{array}{c}\text { Internet interactive } \\
\text { marketing strategy }\end{array}$ & -1.598 & -3.385 & $\begin{array}{c}\text { Internet interactive } \\
\text { marketing strategy }\end{array}$ & -5.005 & -3.134 \\
\hline $\begin{array}{c}\text { message website } \\
\text { strategy }\end{array}$ & -2.546 & -4.323 & $\begin{array}{c}\text { message website } \\
\text { strategy }\end{array}$ & -4.564 & -3.123 \\
\hline $\begin{array}{c}\text { mobile search } \\
\text { strategy }\end{array}$ & -2.645 & -3.424 & $\begin{array}{c}\text { mobile search } \\
\text { strategy }\end{array}$ & -3.563 & -2.983 \\
\hline $\begin{array}{c}\text { mobile business } \\
\text { district strategy }\end{array}$ & -2.674 & -2.865 & $\begin{array}{c}\text { mobile business } \\
\text { district strategy }\end{array}$ & -2.432 & -2.0312 \\
\hline $\begin{array}{c}\text { Wireless website } \\
\text { strategy }\end{array}$ & -2.743 & -3.094 & $\begin{array}{c}\text { Wireless website } \\
\text { strategy }\end{array}$ & -2.64 & -2.093 \\
\hline $\begin{array}{c}\text { Bluetooth interaction } \\
\text { strategy }\end{array}$ & -2.452 & -3.921 & $\begin{array}{c}\text { Bluetooth } \\
\text { interaction strategy }\end{array}$ & -2.533 & -2.031 \\
\hline $\begin{array}{c}\text { QR code scanning by } \\
\text { phone }\end{array}$ & -2.255 & -3.255 & $\begin{array}{c}\text { QR code scanning } \\
\text { by phone }\end{array}$ & -2.032 & -1.3654 \\
\hline $\begin{array}{c}\text { Consumer } \\
\text { satisfaction }\end{array}$ & -2.021 & -2.698 & $\begin{array}{c}\text { Consumer } \\
\text { satisfaction }\end{array}$ & -2.685 & -1.265 \\
\hline $\begin{array}{c}\text { Behavior of } \\
\text { consumers }\end{array}$ & -3.665 & $\begin{array}{c}\text { Behavior of } \\
\text { consumers }\end{array}$ & -2.698 & -2.011 \\
\hline
\end{tabular}


Table 4 Inspection of Residual Sequence e

\begin{tabular}{|c|c|c|c|}
\hline Variable & ADF value & $10 \%$ critical value & $5 \%$ critical value \\
\hline Residual Sequence e1 & -0.246 & -1.623 & -0.122 \\
\hline Residual Sequence e2 & -0.325 & -1.125 & -0.102 \\
\hline
\end{tabular}

Table 5 Multivariate Regression Estimates of the Relationship between Mobile Internet Interactive Marketing and Consumer Behavior

\begin{tabular}{|c|c|c|c|c|}
\hline & coefficient & Standard error & T value & P value \\
\hline constant term & 12.358 & 0.256 & 5.365 & 0.000 \\
\hline message website strategy & 0.258 & 0.657 & 6.255 & 0.000 \\
\hline mobile search strategy & 0.125 & 0.365 & 2.356 & 0.025 \\
\hline $\begin{array}{c}\text { mobile business district } \\
\text { strategy }\end{array}$ & 0.326 & 0.235 & 3.256 & 0.000 \\
\hline $\begin{array}{c}\text { wireless website strategy } \\
\text { bluetooth interaction } \\
\text { strategy }\end{array}$ & 0.214 & 0.364 & 6.2554 & 0.001 \\
\hline $\begin{array}{c}\text { OR code scanning by } \\
\text { phone }\end{array}$ & 0.023 & 0.025 & 5.235 & 0.001 \\
\hline $\mathrm{R}^{2}$ & 0.357 & 0.0365 .365 & 2.8674 & 0.006 \\
\hline $\mathrm{Adj}^{2}$ & \multicolumn{4}{|c|}{0.7658} \\
\hline $\mathrm{F}(\mathrm{P})$ & \multicolumn{4}{|c|}{0.7435} \\
\hline
\end{tabular}

Table 6 Multi-Regression Estimation Results of Relationship between Mobile Internet Interactive Marketing and Consumer Behavior

\begin{tabular}{|c|c|c|c|c|}
\hline & Coefficient & Standard error & T value & P value \\
\hline Constant term & 0.174 & 0.117 & 1.485 & 0.3412 \\
\hline $\begin{array}{l}\text { message website } \\
\text { strategy }\end{array}$ & 0.188 & 0.084 & 2.239 & 0.0634 \\
\hline $\begin{array}{c}\text { mobile search } \\
\text { strategy }\end{array}$ & 0.154 & 1.23342 & 4.673 & 0.0010 \\
\hline $\begin{array}{l}\text { mobile business } \\
\text { district strategy }\end{array}$ & 0.603 & 0.111 & 14.379 & 0.0008 \\
\hline $\begin{array}{l}\text { wireless website } \\
\text { strategy }\end{array}$ & 0.313 & 0.122 & 2.754 & 0.0053 \\
\hline $\begin{array}{l}\text { bluetooth interaction } \\
\text { strategy }\end{array}$ & 0.076 & 0.139 & 13.547 & 0.0009 \\
\hline $\begin{array}{c}\text { code scanning by } \\
\text { phone }\end{array}$ & 0.374 & 0.117 & 1.485 & 0.3412 \\
\hline $\mathrm{R}^{2}$ & \multicolumn{4}{|c|}{0.7256} \\
\hline $\operatorname{Adj}^{2}$ & \multicolumn{4}{|c|}{0.7124} \\
\hline $\mathrm{F}(\mathrm{P})$ & \multicolumn{4}{|c|}{$245.35(0.000)$} \\
\hline
\end{tabular}

\subsection{Impact of price changes on consumer demand for purchasing behavior}

When other factors, such as the cost of information acquisition, remain constant, price factors have become the main consideration for consumers to choose brands of Internet finance industry. As shown in Figure 1, when the acquisition cost $\mathrm{C}(\mathrm{E})$ of brand information is kept constant $\mathrm{C} 0(\mathrm{E})$, the initial consumer demand curve is D0, the corresponding price is P0, and the demand quantity is Q0. When the price rises, the demand of consumer will inevitably decrease owing to the increased cost of consumption, and the demand curve will move to the left, ie D in the figure. When moving to D1, the corresponding demand decreases from Q0 to Q1. When prices fall, consumers' demand will inevitably increase due to the decreased costs of consumption. Therefore, the demand for the Internet 
financial industry brand products increased, and the demand curve moved to the right. That is, D0 moves to D2, and the corresponding demand increases from Q0 to Q2.

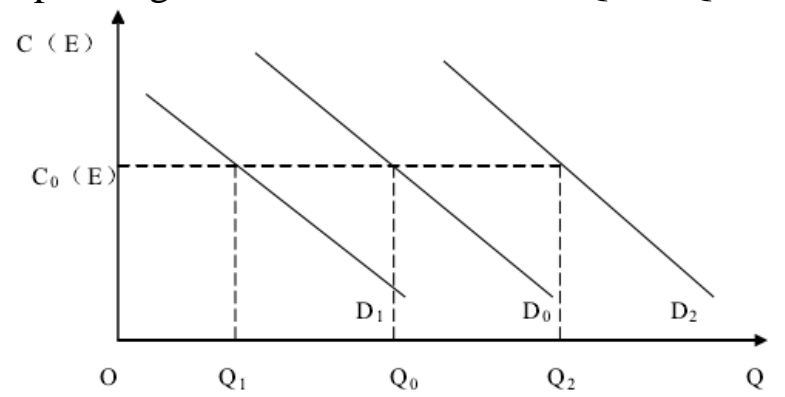

Figure 1 Effect of Price Change on Demand under Constant Information Acquisition Cost

\section{Conclusion}

First, the cointegration test yields first-order stability between the internet interactive marketing strategy, its six dimensions, and the consumer behavior variables. Second, the multiple regression estimate of the relationship between mobile internet interactive marketing and consumer willingness draw a conclusion that the estimation coefficients of mobile internet company SMS website strategy, mobile search strategy, mobile shopping district strategy, wireless website strategy, Bluetooth interaction strategy, and mobile phone two-dimensional code scanning estimation are respectively $0.258,0.125,0.326,0.214,0.023$, and 0.357 . There is an positive correlation among the mobile Internet enterprise SMS website strategy, mobile search strategy, mobile shopping district strategy, wireless website strategy, Bluetooth interaction strategy, mobile phone QR code scanning and consumer willingness. Third, the multiple regression of the relationship between mobile internet interactive marketing and consumer behavior draw a conclusion that the estimated coefficients of the mobile Internet company SMS website strategy, mobile search strategy, mobile business district strategy, wireless website strategy, Bluetooth interaction strategy, and mobile phone QR code scanning are $0.188,0.154,0.603,0.313,0.076$, and 0.374 . There is a positive correlation among mobile Internet enterprise SMS URL strategy, mobile search strategy, mobile shopping district strategy, wireless website strategy, Bluetooth interaction strategy, mobile phone QR code scanning, and consumer behavior.

In the Internet environment, China's mobile Internet companies should make full use of the opportunities for interactive marketing of the Internet, and actively use the SMS website strategy, mobile search strategy, mobile shopping strategy, wireless website strategy, Bluetooth interaction strategy, mobile phone QR code scanning to enhance and consume the interaction between individuals and promote consumers' understanding and communication of products and services, so as to enhance consumer experience, repurchasing behavior and loyalty of corporate brands, and inspire consumers to promote it to others.

\section{References}

[1] Shang Zihui. Study on the Influence of Tea Culture Promotion Information on Consumers' Purchase Intention in the Internet+Era [J].Fujian Tea, 2018(07):46.

[2] Impressing New Middle School with Recessive Values [J]. Business School, 2018(06): 84-86.

[3] Ding Zhaoqiao. Research on the Main Operational Model of Cross-border E-Commerce of Enterprises [J]. Journal of Jiangsu Vocational and Technical College of Commerce, 2018(03): 17-21.

[4] Su Yong, Fang Lingzhi, Chen Yunyong. The formation and expansion of brand emotions-- A survey based on emotional marketing [J/OL]. China's circulation economy, 2018(06):53-61[2018-06-13].https://doi.org/10.14089/j.cnki.cn11-3664/f.2018.06.006.

[5] Zu Ming, Yang Wu. Research progress on the influencing factors of purchase of new energy vehicles [J].Green Technology, 2018(10):241-244+260. 